\title{
Warm calibration unit of the mid- infrared E-ELT instrument METIS: overview and current status of the project
}

Rutowska, Monika, Sharma, Tarun, Wiest, Michael, Graf, Sabine, Straubmeier, Christian, et al.

Monika Rutowska, Tarun Sharma, Michael Wiest, Sabine Graf, Christian Straubmeier, Steffen Rost, Lucas Labadie, Andreas Eckart, Leonard Burtscher, Tibor Agócs, Dirk Lesman, Remko Stuik, Adrian Glauser, Bernhard Brandl, Felix Bettonvil, "Warm calibration unit of the mid-infrared E-ELT instrument METIS: overview and current status of the project," Proc. SPIE 11447, Ground-based and Airborne Instrumentation for Astronomy VIII, 114472T (13 December 2020); doi: 10.1117/12.2562219 


\title{
Warm calibration unit of the mid-infrared E-ELT instrument METIS - overview of current status of the project
}

\author{
Monika Rutowska ${ }^{a}$, Tarun Sharma ${ }^{\mathrm{a}}$, Michael Wiest ${ }^{\mathrm{a}}$, Sabine Graf ${ }^{\mathrm{a}}$, Christian Straubmeier ${ }^{\mathrm{a}}$, \\ Steffen Rost ${ }^{\mathrm{a}}$, Lucas Labadie ${ }^{\mathrm{a}}$, Andreas Eckart ${ }^{\mathrm{a}}$, Leonard Burtscher ${ }^{\mathrm{b}}$, Tibor Agócs ${ }^{\mathrm{c}}$, Dirk \\ Lesman $^{\mathrm{c}}$, Remko Stuik ${ }^{\mathrm{b}}$, Adrian Glauser ${ }^{\mathrm{d}}$, Bernhard Brandl ${ }^{\mathrm{b}}$, and Felix Bettonvil ${ }^{\mathrm{b}}$ \\ a' Physikalisches Institut, Universität zu Köln, Zülpicher Straße 77, 50937, Köln, Germany \\ ${ }^{b}$ Leiden Observatory, Leiden University, P.O. Box 9513, 2300 RA Leiden, The Netherlands \\ ${ }^{\mathrm{c}}$ NOVA Optical Infrared Instrumentation Group at ASTRON, P.O. Box 2, 7990 AA \\ Dwingeloo, The Netherlands \\ ${ }^{\mathrm{d}}$ ETH Zürich, Institute for Particle Physics and Astrophysics, Wolfgang-Pauli-Strasse 27, \\ CH-8093 Zürich, Switzerland
}

\begin{abstract}
The warm calibration unit (WCU) is one subsystem of the future METIS instrument on the European Extremely Large Telescope (E-ELT). Operating at daytime temperature, the WCU is mounted above the main cryostat of METIS and will be employed as calibration reference for science observations, as well as for verification and alignment purposes during the AIT phase. The WCU is designed and constructed at the University of Cologne, partner in the METIS consortium. The WCU, together with the full METIS instrument, went recently through a successful preliminary design review (PDR) phase at ESO and is entering now the Phase $\mathrm{C}$ of the project. In this paper, we present the current status of the WCU and summarize the mostly mechanical and optical engineering work. We adopted a hexapod unit to interface with the METIS cryostat and a CFRP-based optical bench to optimally cope with alignment flexure. We develop the case for fiber-fed laser sources feeding the integrating sphere for spectral calibration of the LM-Spectrograph of METIS. We detail the activity foreseen for Phase C including the optical tolerances analysis, the eigenfrequency and earthquake analysis and a preparation of the sub-system MAIT work, finishing the paper with a short overview of the WCU future plans.
\end{abstract}

Keywords: E-ELT, METIS, Warm Calibration Unit, mid-infrared, hexapods, alignment, calibration, AIT

\section{INTRODUCTION}

The METIS instrument is a part of the first-light instrument suite that will equip the new E-ELT towards the end of 2020s. The other first-light instruments are MICADO and HARMONI dedicated to high-resolution imaging and spectroscopy in the near-infrared spectral range. The MAORY instrument module will implement Multi-Conjugate Adaptive Optics to deliver to MICADO a wide field-of-view corrected from the atmospheric turbulence. METIS will be the only instrument to observe in the mid-infrared range, ${ }^{1}$ from $3 \mu \mathrm{m}$ to $13 \mu \mathrm{m}$, and deliver diffraction-limited imaging and high-resolution spectroscopy simultaneously in this wavelength range. The science covered by METIS will be very diverse, ranging from the study of nearby exoplanets and protoplanetary disks to the study of high-red shift galaxies and the Galactic Center. It will be an ideal complement to existing or upcoming facilities like ALMA ${ }^{2}$ or the James Webb Space Telescope. ${ }^{3}$ METIS is built by a large consortium of European research institutions led by NOVA in the Netherlands with each partner responsible for a sub-system of the instrument. The full assembly and integration of the instrument will take place in Leiden before shipment to Chile, at the summit of Cerro Armazones.

Further author information: (Send correspondence to Monika Rutowska)

E-mail: rutowska@ph1.uni-koeln.de, Telephone: 00492214707791

Ground-based and Airborne Instrumentation for Astronomy VIII, edited by Christopher J. Evans,

Julia J. Bryant, Kentaro Motohara, Proc. of SPIE Vol. 11447, 114472T · C 2020

SPIE · CCC code: $0277-786 X / 20 / \$ 21 \cdot$ doi: $10.1117 / 12.2562219$

Proc. of SPIE Vol. 11447 114472T-1

Downloaded From: https://www.spiedigitallibrary.org/conference-proceedings-of-spie on 25 Jan 2021 
One sub-system of METIS is the so-called Warm Calibration Unit (WCU) developed at the University of Cologne. The main role of the WCU is to supply METIS with functionalities able to test, calibrate and troubleshoot the METIS instrument as a whole. In this sense, several artificial sources will be provided by the WCU to calibrate the detector response, to test the delivered image quality and understand the performances of the high-spectral resolution LMS subsystem. This paper describes the conceptual principle of the WCU and details the adopted design in view of the next important milestone for the METIS project, namely the Final Design Review planned in July 2022.

\section{OVERVIEW OF WARM CALIBRATION DESIGN}

\subsection{General overview: Warm Calibration Unit in METIS}

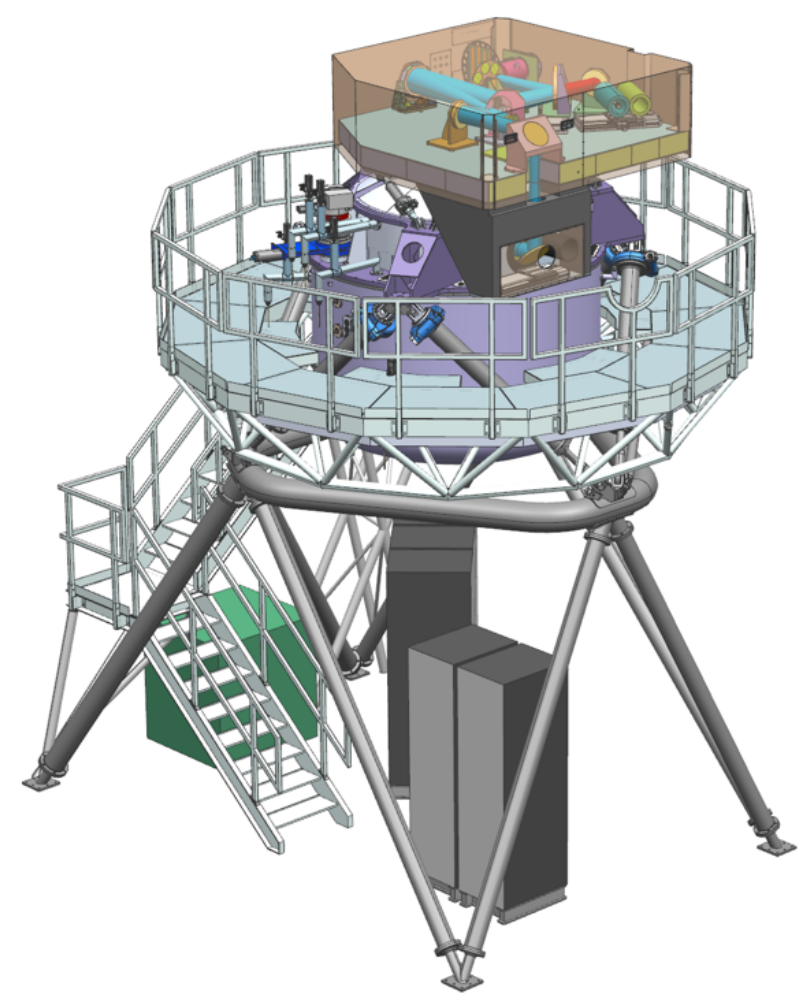

Figure 1. A scheme overview of the current METIS design. At the top of METIS is the WCU and below warm support structure (WSS) and the cryostat. Next to the stairs are electrical cabinets (gray cuboids). One green cuboid indicates a space reserved for the WCU laser box. Enclosure of the WCU is transparent and a blue cylinders indicate here an optical beam coming out the blackbody source (pink cylinder in a top of corner of the WCU) through a periscopic arm into METIS.

The functional layout of the WCU is schematically shown in Figure 2 and was presented at SPIE2018 conference by N. Baccichet. ${ }^{4}$ The WCU is represented by the pink envelope, and it is attached on the top part of METIS, outside the main cryostat around $7 \mathrm{~m}$ height from the Nasmyth platform. Six linear actuators building together hexapods connect WCU to the cryostat structure below by connecting to the stiff ring present on the top part of the cryostat. A work progress and current design of the hexapod we describe in subsection: 2.3. The main part of WCU is an optical bench made of a carbon fiber reinforced polymer (CFRP). The optical bench holds the WCU focal plane sources and relay optics. The optical beam is shown by a blue beam in Figure 1 .

The WCU light is fed into the cryostat by means of a periscopic arm placed between its entrance window and the E-ELT focal plane (indicated by a red arrow in Figure 2). The periscope arm is equipped with a linear stage used to select two different feeding modes: a mirror, which reflects light from a top part of WCU into 


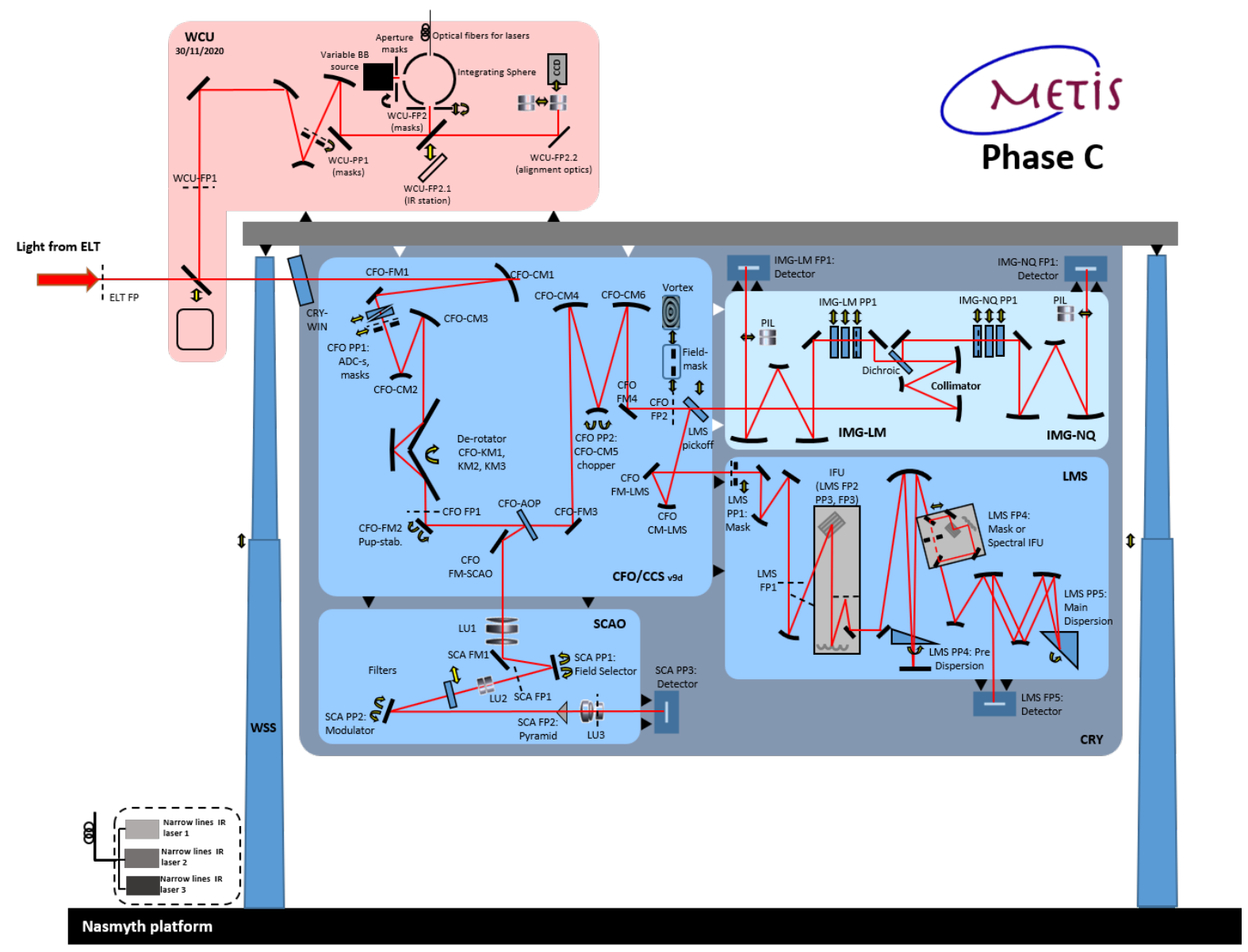

Figure 2. Schematic view of the METIS instrument. The WCU is positioned above the cryostat. The laser box is placed at Nasmyth platform.

METIS window when WCU is 'in-use'. When not 'in-use', the WCU can be closed by a sealing cylinder that can be moved around the incoming E-ELT beam. Details of optical setup and tolerances analysis are presented in subsection: 2.4 and 2.5 .

One of the important issues, which METIS had faced during PDR review was the total weight. All of subsystems took the numbers of actions in order to reduce its weight. The WCU has given up a plan of adopting a double enclosure with a complex cooling system. Instead of that we propose a single, light weight enclosure and reduce temperature coming out of a potential heat sources locally inside WCU. In addition, the monochromatic sources from the main structure of WCU were removed. This action is in detail presented in subsection: 2.6. For final design of the WCU we aim to keep the total weight of the WCU as $1300 \mathrm{~kg}$ and the WCU laser box as $200 \mathrm{~kg}$.

\subsection{The functionalities of the WCU}

The WCU provides a number of different functionalities for METIS. Mainly, the WCU is divided in two focalplane stations, one to carry out tasks in the mid-IR range, and a second one designed for specific alignment and monitoring functions at visible wavelengths, called WCU-FP2.1 and WCU-FP2.2 respectively. ${ }^{4}$ A broadband light source as the blackbody (BB) has been selected for a stable source flux. In addition, the aperture masks (indicated in Figure 2) can adjust a flux level in a relatively short time. The flux adjustment leads to a detector linearity tests. The BB with a combination of the integrating sphere (IS) set up a flat-fielding calibration. Adding a focal plane masks (single point and multiple) in the front of IS output (shown as WCU-FP2 masks in Figure 2) provides a high-Strehl ratio point sources and image quality measurements. Then, three spherical mirrors generate Offner Relay optics and here with accessible pupil plane masks (shown as WCU-PP1 masks 
in Figure 2) reconstruct precisely E-ELT beam characteristic. Thanks to monochromatic sources in WCU laser box a wavelength calibration of LMS can be proceeded.

Optical lab tests and investigations of a stable source flux as the BB, flat-fielding and flux adjustment with a use of the aperture masks are presented in this SPIE2020 conference by Sabine Graf in the proceedings: "The Warm Calibration Unit of METIS - Laboratory tests and proof-of-concept".

\subsection{The optical bench and supporting hexapods}

As it was not discussed in this paper the WCU will be in operation only during daytime. Operational temperature range is from $0^{\circ} \mathrm{C}$ to $15^{\circ} \mathrm{C}$. No ambient temperature inside WCU is foreseen and no thermally resistant enclosure. It means at one side, the WCU should be not warmer or colder than $\pm 2.5^{\circ} \mathrm{C}$ in a comparison to temperature inside the E-ELT dome. At other side, it means that the WCU may be effected by any temperature differences within E-ELT dome. In order to reduce any thermal effects all WCU elements are expected to be made of a very low CTE materials and matching between each other as close as possible. Following this and weight restrictions it is planned that WCU bench is made of CFRP material.

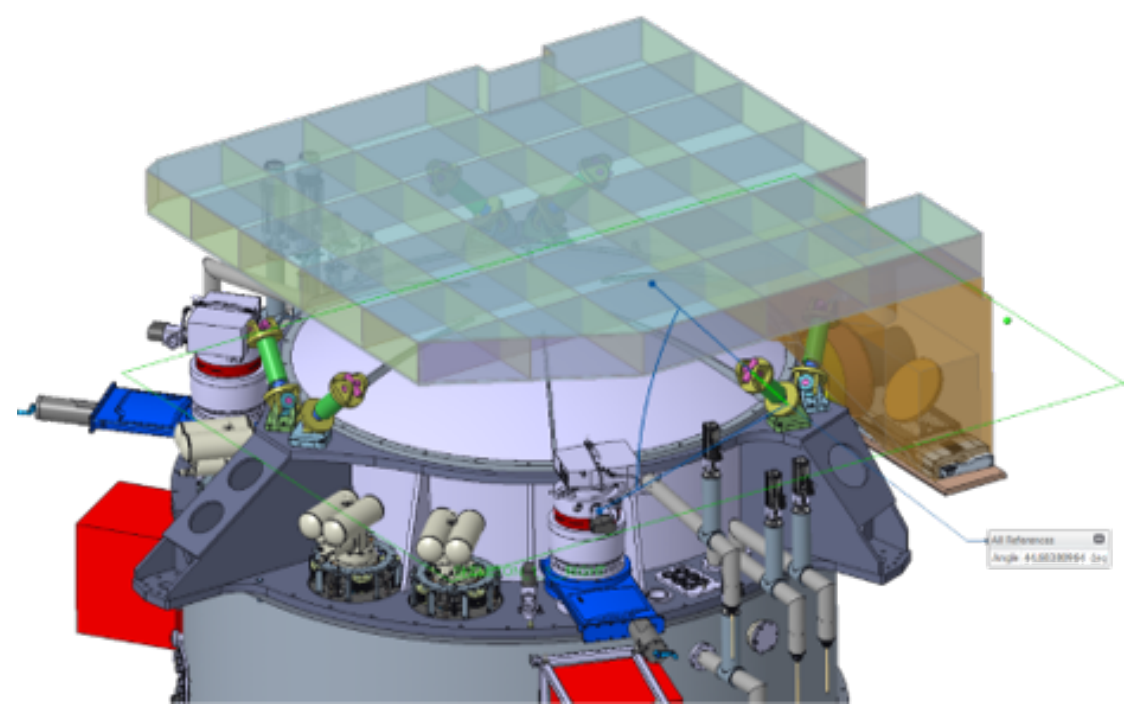

Figure 3. A scheme of the WCU optical bench with a preliminary designed ribs within the bench and supported by 'short' hexapods.

Figure 3 presents the WCU optical bench. This design was further taken into the gravity loads modeling using finite element methods analysis. Inside the bench are ribs and carbon fibers. At top and bottom are two cover-plates. The size of the bench is $20 \mathrm{~cm} \times 250 \mathrm{~cm} \mathrm{x} 250 \mathrm{~cm}$. The estimated weight is $260 \mathrm{~kg}$. CTE of the covers is estimated as: $1.0 \mathrm{E}^{-6} \mathrm{~mm} / \mathrm{mm} \cdot \mathrm{K}$ and CTE of the ribs is $1.3 \mathrm{E}^{-6} \mathrm{~mm} / \mathrm{mm} \cdot \mathrm{K}$. A periscope arm structure is foreseen to be made out of CFRP material, too. And as one element with WCU bench.

Figure 4 (left side) shows three brackets (green color) indicating attachment for six hexapods (F1-F6). Figure 4 (right side) shows two pairs (F1-F2) and (F5-F6) of the hexapods attached to lower part of the cryostat. For a comparison, Figure 3 shows two pairs of the hexapods attached to upper part of the cryostat. This design of the hexapod interfaces was dictated by a good and easy access to PTC coolers at the cryostat structure (double, white cylinders at Figure 3). However, it can be noticed that the hexapods in Figure 3 have a large opening angle and we can find them as in 'horizontal' position. These hexapods we labelled as 'short' ones.

One of the main functions of the hexapods is alignment of the WCU to CFO focal and pupil planes. This drives travel range requirements as: $\pm 26 \mathrm{~cm}$ in x-axis, \pm 25 in y-xis, and \pm 55 in z-axis, and rotational axis: $\pm 1.7^{\circ}$ in $\mathrm{x}$-axis, $\pm 1.6^{\circ}$ in $\mathrm{y}$-axis, $\pm 1.6^{\circ}$ in z-axis for the hexapods. It was calculated that 'short' hexapods cannot fully cover the required travel range. We propose to extend the hexapods length, reduce opening angle and attach the hexapods at a lower part of the cryostat. At the right side of Figure 4 are shown the 6 hexapods design (labeled 'long'). These hexapods are symmetric and can cover all required travel range. Moreover, the hexapods 

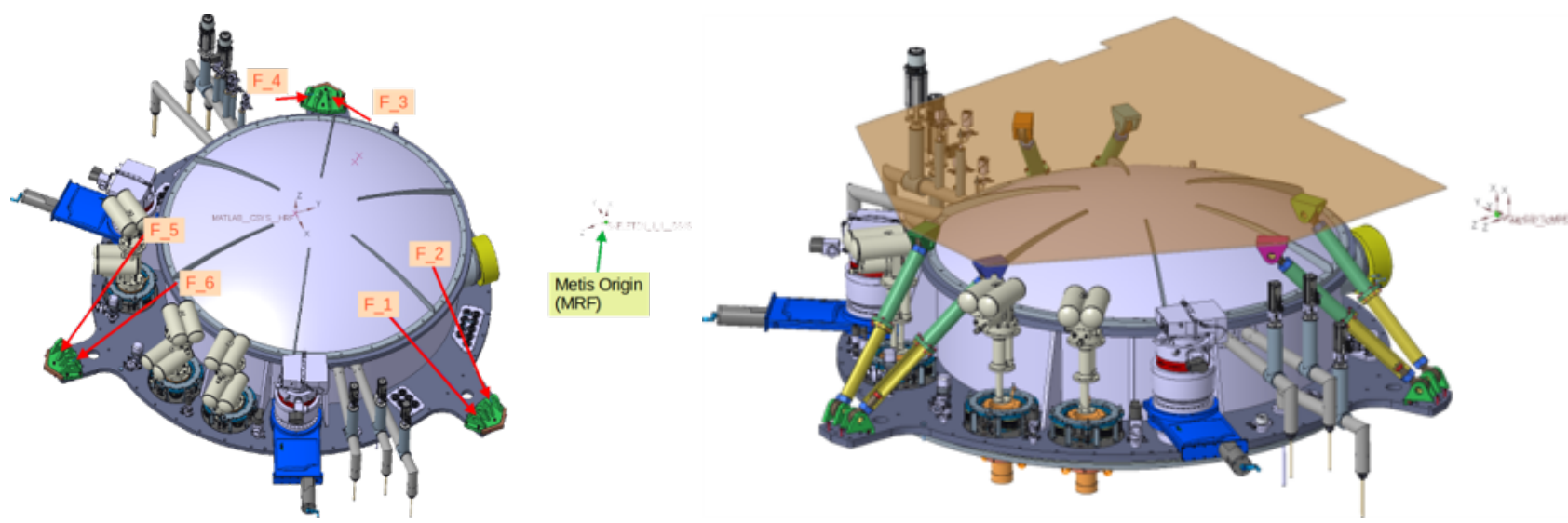

Figure 4. A top CAD overviews of a part of the cryostat, at the left side is shown WCU/CRY interface (green colors) and at the right side the 'long' hexapod design

accuracy shall be in the range $\pm 330 \mu m$ and the repeatability $\pm 49 \mu m$ in order to align the WCU focal and WCU exit pupil planes to CFO focal and exit pupil planes, respectively. The new 'long' hexapods satisfies these requirements, too. It is under investigations the access to PTC. The left side of Figure 4 shows that PTCs next to F5 and F6 hexapods can be rotated. It may help to maintain or remove PTCs.

\subsection{The Offner optical relay}

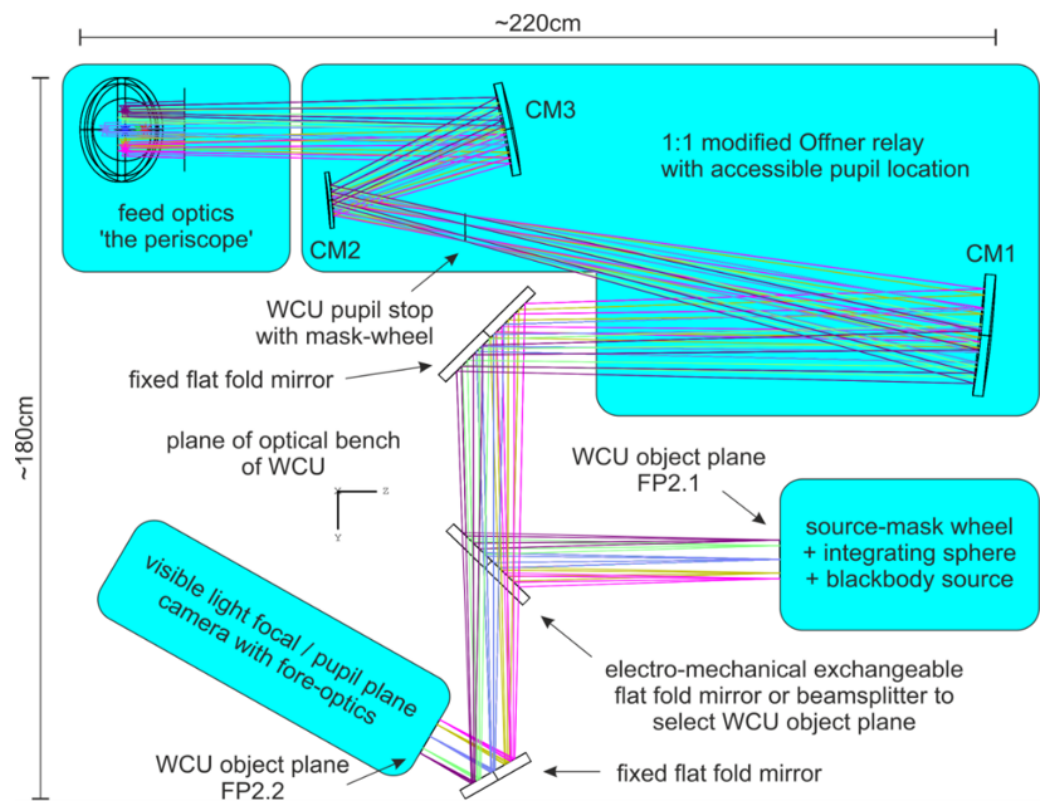

Figure 5. ZEMAX layout of the optical design of the WCU in y-z plane (i.e. when you see METIS from the top with E-ELT to your left.

As was written in the above subsection: 2.2 the main light source in WCU is the blackbody source. The BB beam is equally scattered within the integrating sphere, and can be adjusted by focal plane masks. It is pointed at Figure 5 as WCU object plane FP2.1. The beam can be splitted with a use of the beam splitter attached onto a linear stage and partly forwarded into 'alignment optics'. If the beam splitter is not set at the optical path of the BB beam, the first flat fold mounted at $90^{\circ}$ mirror is placed there. This mirror reflects the beam into the second flat mirror and directs the beam into Offner Relay optics. It is a setting of three spherical mirrors: two 
concave and one convex mirrors. Between CM1 and CM2 is a pupil plane. At the pupil plane is foreseen to have a linear and rotation stage for a pupil plane masks. The beam leaves Offner Relay optics and it is reflected by the sixth mirror: flat fold down mounted at $45^{\circ}$ to the table. This mirror is the last one attached onto WCU bench. It directs the beam into the WCU periscope arm. At some part of the periscope arm is a virtual focal plane WCU FP1 (indicated in Figure 2). At the bottom of the periscope arm is a linear stage with the last, seventh flat mirror called: periscope fold. It is mounted at $45^{\circ}$ to the stage. It reflects the shaped WCU beam into METIS window. The shaped beam has exact characteristic as E-ELT beam. The focal number (F\#) 17.75, field of view (FoV) 29" and circular shape.

All seven mirrors are planned to be made of Zerodur, which has a good reflections properties for infrared wavelengths. The choice of Zerodur is dictated by a low CTE value of this glass. In addition, the mirrors will be coated by a protected gold.

\subsection{Optical tolerances analysis}

The optical tolerances analysis has been completed for the set of 7 mirrors. Using ZEMAX tolerances module and Monte Carlo 20.000,00 cycles we analyzed a number of different scenarios for a static conditions as: separation between mirrors surface $100 \mathrm{~mm}$, tolerance on a curvature of spherical mirrors: $0.1 \%$, tip/tilt error: 0.023 , mechanical tolerances: $60 \mu \mathrm{m}$, surface form error per mirror: $15 \mathrm{~nm}$. With a number of combinations of described parameters and run simulations we gain a strong confidence that with the presented optical setup WCU fulfills the optical system requirements as F\# in the range: $17.67-17.83$, total WFE: $109 \mathrm{~nm}$, exit pupil location at $37868 \mathrm{~mm} \pm 78 \mathrm{~mm}$ and Strehl ratio for wavelength1.45 $\mu \mathrm{m}$ is greater than $80 \%$.

Moreover, the first analysis of effect of the gravity loads and its consequences in the mirrors' deformation have been analyzed. For that, a simplified model of WCU has been uploaded into COMSOL Multiphysics Software and AutoCad Inventor. A simplified model of WCU contain a new design of mirrors' mounts. The mirrors mounts design is not fully completed, still details of attachment of the mirror into the mount is an open. The center of mirrors are at optical path at $30 \mathrm{~cm}$ height from the bench surface. A concept of these mirrors mounts design is shown in Figure 6. Here, we present 4 mirrors mounts for CM1, CM2, F1 table fold flat mirror, the periscope fold mirror, respectively. The mounts are planned to be made of INVAR. Another material of low CTE. One of disadvantage of INVAR is its weight. For this reason the mounts structure is not solid, but having lots of triangular shape cuts-off. Other side, the mounts need to be as stiff as to survive the earthquake loads.
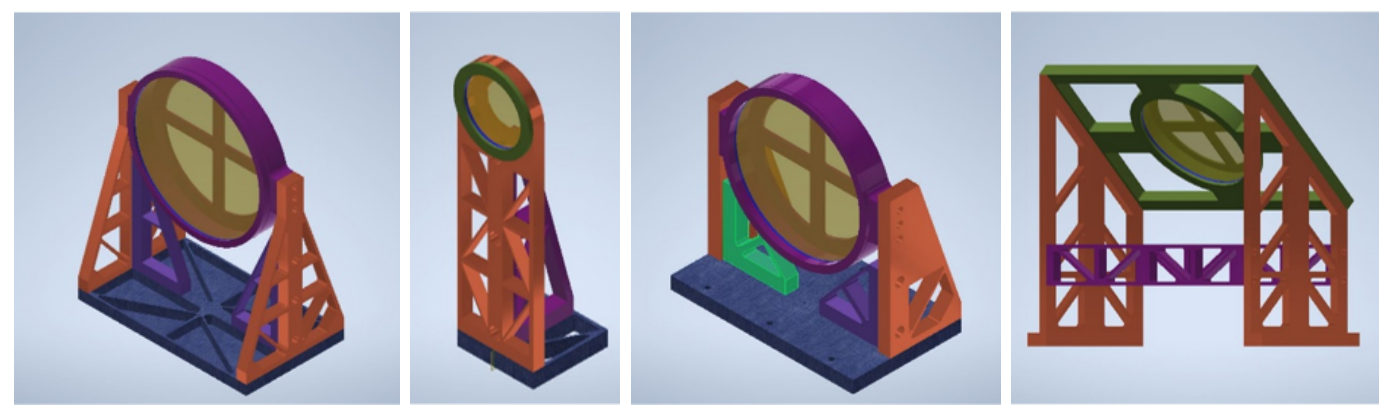

Figure 6. The four mirrors' mounts concept design as for CM1 mirror (the largest spherical one), CM2 mirror (the smallest spherical one), the first table fold flat mirror and the periscope fold, respectively.

Figure 7 presents the results for the gravity deformation analysis. In these three figures we see a simplified WCU bench and its components, and displacement of each component in X-position, Y-position, Z-position. The gravity loads is applied in a vertical direction. Each of displacement components was translated into ZEMAX tolerance analysis as: displacement of center of components for $\mathrm{X}, \mathrm{Y}, \mathrm{Z}$ axes. In the case of decentre of components for vertical shifts the range is: $-222 \mu \mathrm{m}$ up to $+89 \mu \mathrm{m}$ and for lateral shifts the range is: $-38 \mu \mathrm{m}$ up to $+15 \mu \mathrm{m}$. A changed distance between components was recounted in the range of $12 \mu \mathrm{m}$ up to $53 \mu \mathrm{m}$. And the last effected parameter, which was applied into ZEMAX was tilt of components in $\mathrm{x}$-axis and $\mathrm{y}$-axis in the range 0.00014 degree to 0.00094 degree. As we can observe that at Figure 7 always the periscope mirror is the mostly affected by the gravity. For this mirror are the lowest values as $-222 \mu \mathrm{m}$. 


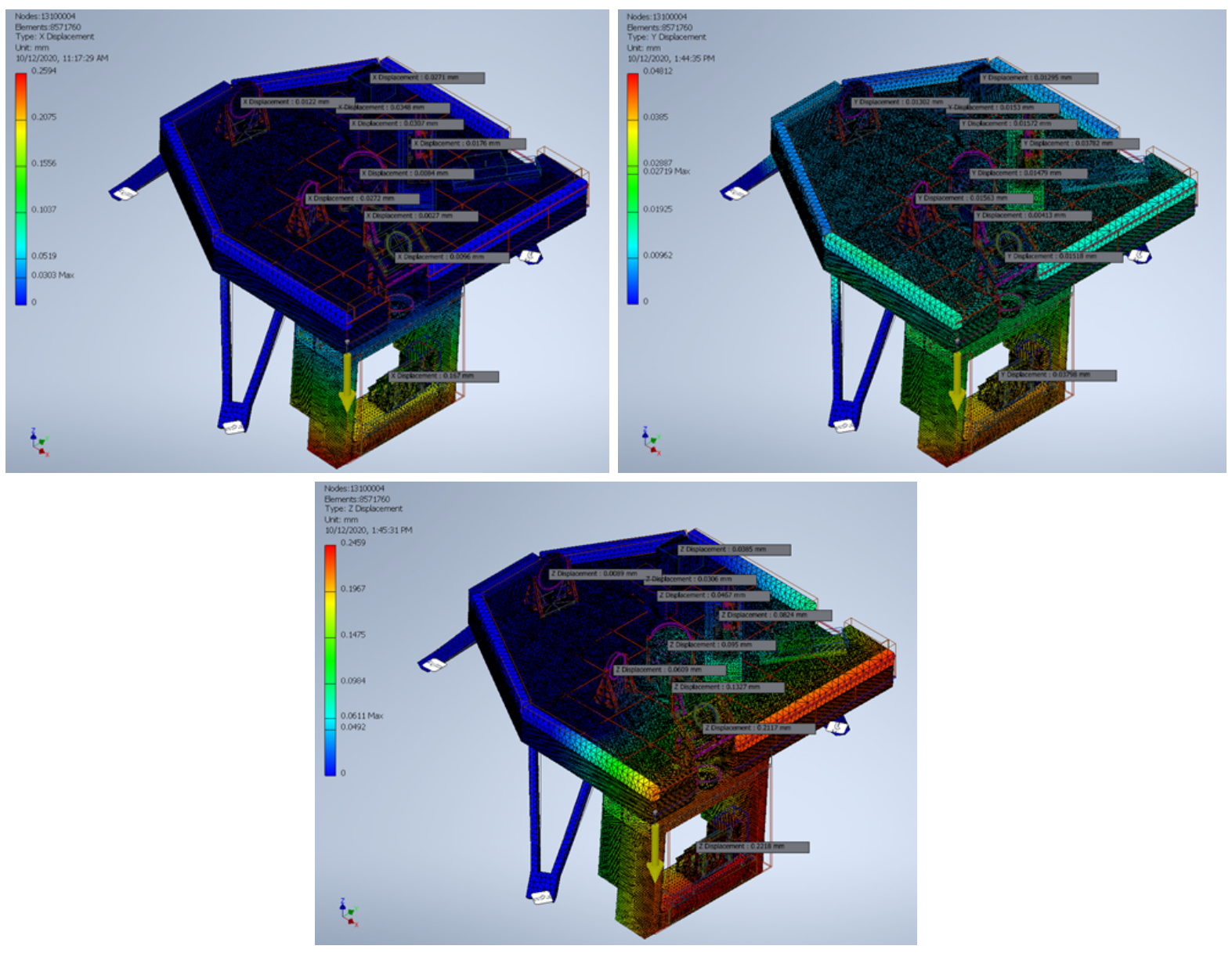

Figure 7. A displacement of all the optical components on the WCU bench in X-position, Y-position, and Z-position due to a vertically applied gravity loads.

Even if all these displacements, changed distances and extra tilts are taken into calculations still the WCU optical setup is compliant to its optical requirements.

\subsection{The spectral sources in WCU}

As was presented ${ }^{4}$ it was proposed to employ tunable QCLs lasers within operating range: $3.8 \mu \mathrm{m}$ to $11.0 \mu \mathrm{m}$ in order to calibrate long-measurement spectrograph (LMS) and high-contrast imager (HCI). However, a daily wavelength calibration of METIS is planned to be completed using a ELT sky-observation after AIT/AIV phases. Covering a full operations METIS range by lasers is no longer overseen. In this paper we propose to use three single lines lasers only for AIT and AIV phase of the project. And later as the emergency control of the system for example after earthquake.

\subsubsection{The WCU laser box}

The monochromatic sources (as lasers) are no longer inside the main WCU structure and attached to the CFRP bench. The lasers and its items are a separate unit named: the WCU laser box. It is located on the Nasmyth platform and connected to the WCU by the optical fibers. The WCU laser box will be placed below METIS, next to the stairs quite close to ICS cabinets. Figure 1 shows a reserved space for the WCU laser box as a green cuboid close to the stairs. It was previously discussed to attach the WCU laser box onto RIG platform or for example at some parts of WSS structure. Taking into consideration: access to the lasers, safety aspects and complication of the design it has been concluded that the Nasmyth platform is the best choice. It is planned to 
have three lasers, as it is drawn in Figure 2. Details of the lasers are described in lower subsections. The lasers and its items can be placed into a temperature and humidity controlled cabinet. The cabinet keeps ambient temperature inside, that it is crucial for a stable lasers' operations. At the cabinet will be an electrical panel in order to control the lasers' by ICS software and the optical fibers interfaces.

\subsubsection{MIDIR lasers}

Having light sources covering infra-red region and which can be used for the wavelength calibration is still quite limited at the market. The most of calibration lamps generate wavelengths shorter than $2.5 \mu \mathrm{m}$. The lasers choices are limited, too. One of laser, which covers for example the range of $1.45 \mu \mathrm{m}$ to $4.00 \mu \mathrm{m}(300 \mathrm{GHz}$ tuning range and the linewidth less than $2 \mathrm{MHz}$ ) is Toptica laser: singly-resonant optical parametric oscillator laser source (TOPO). Then, there are numbers of tunable quantum cascade lasers from $4 \mu \mathrm{m}$ up to $11 \mu \mathrm{m}$. For example combination of 4 MIRcat QCLs chips of DayLight Solution in a single housing can cover the wavelength range from $4.1 \mu \mathrm{m}$ to $5.3 \mu \mathrm{m}$. And for example, 4 QCLs within N-band can cover range from $6.0 \mu \mathrm{m}$ to $11 \mu \mathrm{m}$. A typical spectral linewidth is less than $1 \mathrm{~cm}^{-1}$. However, pulsed and CW tunable QCLs have a quite poor performance for operating in the range of wavelengths shorter than $4.00 \mu \mathrm{m}$. These lasers: TOPO and tunable QCLs have two common factors: the cost and a high power. There are an expensive lasers and too powerful for METIS. For our project, it has been agreed to use the lasers / IR light sources, which operates within the region from $3.00 \mu \mathrm{m}$ to $5.50 \mu \mathrm{m}$ to calibrate the LMS spectrograph. However, only during AIT and AIV phase. Then, a need to employ such expensive lasers (as TOPO and tunable QCLs) is not justify any longer. We have decided to limit lasers' quantity as much as was possible. Together with calibration scientists, instrumentation engineers, and LMS team it has been chosen to employ only three single lasers lines to cover a blue end of LMS as $3.00 \mu m-3.50 \mu m$, and a red end of LMS as $5.00 \mu m-5.50 \mu m$. In addition, CO fundament transition at $4.65 \mu \mathrm{m}\left(2148 \mathrm{~cm}^{-1}\right)$ is very import line. A precise calibration around this line is in the best interest of a whole METIS team.

\subsubsection{Blue end lasers}

Finding a single line with enough narrow linewidth as $0.2 \mathrm{~cm}^{-1}$ at $3.00 \mu m-3.50 \mu \mathrm{m}$ is quite challenging and it is still under investigations. One of our nowadays proposition is Newport, HeNe, CW laser at $3.39 \mu m$. However, the maximum power of this laser is $7 \mathrm{~mW}$. We have already started a number of lab tests of this laser. The HeNe beam is focused by $150 \mathrm{~mm}$ FL lens into a hollow-core, $500 \mu \mathrm{m}$ core diameter, optical fiber (the fibers' specifications is described in the subsection below). Unfortunately, due to the corona lock-downs these lab tests are delayed and the first transmission results will be not presented in this paper. Another, the laser source which is under consideration as 'the blue end laser' is a single spatial mode and longitudinal mode, distributed feedback interband cascade laser (DFB QCL Thorlabs ID3250HHLH) operating within the range of $3.00 \mu \mathrm{m}-3.50 \mu \mathrm{m}$ with a central wavelengths at $3.28 \mu \mathrm{m}$. The advantage of this laser is that lines can be tuned with the range of $2 \mathrm{~cm}^{-1}$ due to temperature and current changes. Here, we would have a few lines of the blue end instead of one, and shorter than $3.39 \mu \mathrm{m}$. However, this laser is even less powerful and generates the lines with a typical power of $5 \mathrm{~mW}$.

\subsubsection{CO fundamental transitions}

In order to deliver the beam centered at $4.65 \mu \mathrm{m}$, with a spectral linewidth $0.2 \mathrm{~cm}^{-1}$, we are proposing DFB QCL Hamamatsu laser at exactly center peak as $4.65 \mu \mathrm{m}$ (L12004-2190H-E). This laser is a custom-order laser. And as DFB QCL centered peak can be tuned by temperature and current adjustment within range by tuning range $\pm 1.0 \mathrm{~cm}^{-1}$ for the temperature and $\pm 0.5 \mathrm{~cm}^{-1}$ for the current. A typical power is around $20 \mathrm{~mW}$. Employing DFB QCLs for the WCU laser box means in parallel that the operating temperature and current have to be monitored simultaneously with a position of the laser peak. It is possible by attaching the laser diode into a high heat load (HHL) mount and TEC controllers as shown in Figure 8. For monitoring the center of peak we plan to split the laser beam, where one part of the beam is collected by a wavemeter and another one is focused by lens or reflected by a mirror into the optical fiber. Figure 8 presents our concept. 


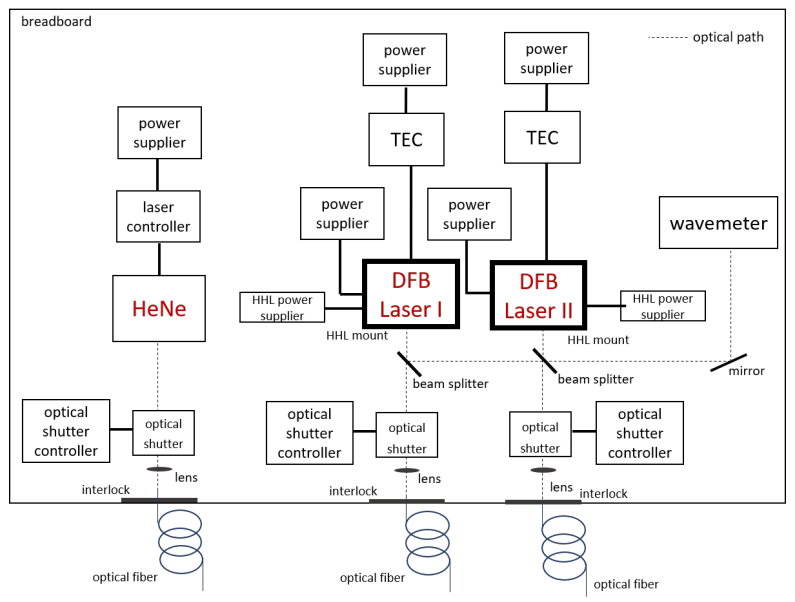

Figure 8. A scheme of lasers and its items inside WCU laser box

\subsubsection{Red end lasers}

For wavelengths longer than $5.00 \mu \mathrm{m}$ a number of available lasers is much higher. It can be pulsed and CW tunable QCLs, single lines DFB QCLs, or even $\mathrm{CO}_{2}$ laser at $5.5 \mu \mathrm{m}$. However, for our project we propose Hamamatsu DFB QCL (L12002-1900H-E) operating at $5.26 \mu \mathrm{m}$, with a spectral linewidth $0.2 \mathrm{~cm}^{-1}$ and tunning range of the temperature $\pm 1.0 \mathrm{~cm}^{-1}$ and of the current $\pm 0.5 \mathrm{~cm}^{-1}$. Similar to above described DFB QCL at $4.65 \mu \mathrm{m}$ and as it is schemed in Figure 8 the laser will be attached to HHL mount and connected to the TEC controller. The beam splitter in the front of the laser head can be supported to a flip mount. When this laser is in a use the beam splitter is 'in' the optical path and the beam is partly directed to the wavemeter. When the DFB laser I is in use, this beam splitter is at 'out' position and allows the beam of DFB laser I to be forwarded to the wavemeter. As one can notice at Figure 8, each of three lasers will have each own power supplier, laser controllers, optics, optical shutter, optical shutter controller and interlocks. For the laser safety purpose the optical path can be covered by baffles.

\subsubsection{Optical fiber connections}

The optical fibers transmit the lasers' signal from the WCU laser box to the WCU main structure. Three fibers, coming out of three specified above lasers, are set together inside one protection tube. The fibers' tube is attached to WSS structure and tracked to the top of the WCU enclosure in a shorter of possible path. At the WCU enclosure, above the WCU integrating sphere is a open entrance, where three fibers are pulled out of the tube. Then, each fiber is connected to the WCU integrating sphere using SMA connector. We propose to use a hollow-core optical fiber, manufactured by OptoKnowledge Company, US. The fiber core is a hollow and its internal side is covered by a dielectric layer: silver-lodide (Agl) and outer side is a reflective silver layer. Around the hollow core are protective layers as glass/plastic capillary and a protective buffer. Such fiber transmits infrared beam with lower loss of the signal in a comparison to a solid core MIDIR fibers. For the above described lasers' and its operation range $(3.39 \mu \mathrm{m}, 4.65 \mu \mathrm{m}$ and $5.26 \mu \mathrm{m})$ we propose to use a hollow-core fiber of $500 \mu \mathrm{m}$ core diameters. It transmits more than $90 \%$ of the signal per $1 \mathrm{~m}$ of the fiber. One of disadvantages of these fibers is a limitation up to $5 \mathrm{~m}$ for manufacturing. Taking into consideration that the top of WCU is more than $7 \mathrm{~m}$ high from the Nasmyth platform WCU will need $2 \times 5 \mathrm{~m}$ of the fibers. The first $5 \mathrm{~m}$ can be connected to the integrating sphere and the second piece of $5 \mathrm{~m}$ fiber into the WCU laser box. The both $5 \mathrm{~m}$ pieces are connected between each other using SMA sleeves. Any fibers' contamination should be avoid and the fibers need to fulfill the safety laser aspects. Moreover, in the case of disconnecting or lifting up the WCU the fibers' connection should be easily accessible by operators.

For a better understanding the fibers' transmission specifications, we plot two transmission scenarios: for the laser input power $7 \mathrm{~mW}$ (the left graph of Figure 9) and for the laser input power $20 \mathrm{~mW}$ (the right graph of Figure 9). It was estimated that total fibers' length varies from $6 \mathrm{~m}$ up to $10 \mathrm{~m}$ with one SMA - SMA sleeve 

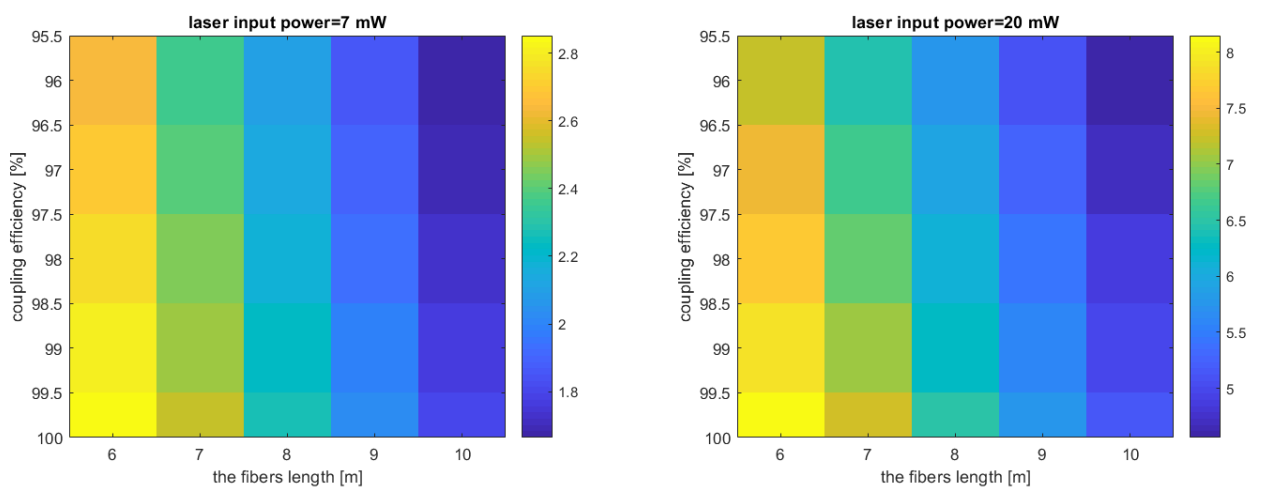

Figure 9. The graphs of laser power transmission for the fibers' length from $6 \mathrm{~m}$ up to $10 \mathrm{~m}$ and the coupling efficiency from $95.5 \%$ up to $100 \%$ for two scenarios: at the left side is laser input power as $7 \mathrm{~mW}$ and at the right side laser input power is $20 \mathrm{~mW}$.

connector. It is estimated that at a 'connection' point a loss of signal equals to $0.8 \mathrm{~dB}$ and the signal loss per fiber is $0.5 \mathrm{~dB} / \mathrm{m}$. In addition, it was added $0.1 \mathrm{~dB}$ loss for example due to bending losses or any losses which may occur. It can be read from the graphs that for $10 \mathrm{~m}$ fibers and $97 \%$ of coupling efficiency, the power for 20 $\mathrm{mW}$ input power drops to $4.7 \mathrm{~mW}$ and for $7 \mathrm{~mW}$ laser drops to $1.7 \mathrm{~mW}$.

\section{CONCLUSIONS}

The conceptual design of the Warm Calibration Unit of METIS and its current status have been presented and its main functions discussed. We have described the mechanical design of the WCU support and the optical bench. As well we have explained the optical setup and discussed static and quasi-static optical tolerances. We have proposed to employ three single lasers lines for the LM spectrograph calibration during AIT/AIV. For the future work we are planning to investigate thermal and vibrations effects on the WCU. Complete the alignment plan and design lifting tools for WCU. Later on, a transportation procedure (from WCU from Cologne, Germany to Leiden, Netherlands) and its requirements needs to be investigated.

\section{ACKNOWLEDGMENTS}

The work described in this paper has been partially supported by the German Federal Department for Education and Research (Bundesministerium für Bildung und Forschung - BMBF) under grant agreement (Verbundforschung) number 05A17PK2.

\section{REFERENCES}

[1] Brandl, B. R., Agócs, T., Aitink-Kroes, G., Bertram, T., Bettonvil, F., van Boekel, R., Boulade, O., Feldt, M., Glasse, A., Glauser, A., Güdel, M., Hurtado, N., Jager, R., Kenworthy, M. A., Mach, M., Meisner, J., Meyer, M., Pantin, E., Quanz, S., Schmid, H. M., Stuik, R., Veninga, A., and Waelkens, C., "Status of the mid-infrared E-ELT imager and spectrograph METIS," in [SPIE proceedings], Evans, C. J., Simard, L., and Takami, H., eds., 9908, 990820 (August 2016).

[2] Wootten, A. and Thompson, A., "The Atacama Large Millimeter/Submillimeter Array," Proceedings of the IEEE 97, 1463-1471 (August 2018).

[3] Gardner, J. P., Mather, J. C., Clampin, M., Doyon, R., Greenhouse, M. a., Hammel, H. B., Hutchings, J. B., Jakobsen, P., Lilly, S. J., Long, K. S., Lunine, J. I., Mccaughrean, M. J., Mountain, M., Nella, J., Rieke, G. H., Rieke, M. J., Rix, H.-W., Smith, E. P., Sonneborn, G., Stiavelli, M., Stockman, H. S., Windhorst, R. a., and Wright, G. S., "The James Webb Space Telescope," Space Science Reviews 123, 485-606 (Novemeber 2006). 
[4] Baccichet, N., Labadie, L., Rost, S., Straubmeier, C., Agócs, T., Jellema, W., Reolfsema, R., van Boekel, R., Glauser, A., Brandl, B., Bettonvil, F., Lynn, J., and Eckart, A., "The calibration unit of the mid-infrared EELT instrument METIS," in [SPIE proceedings,Ground-based and Airborne Instrumentation for Astronomy VII], Evans, C. J., Simard, L., and Takami, H., eds., 1070291, 990820 (July 2018). 